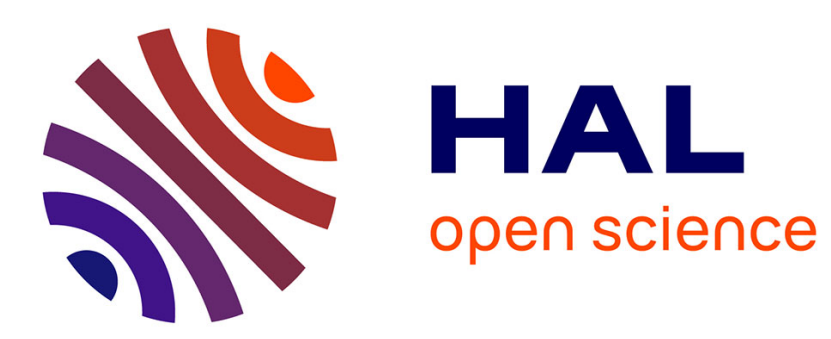

\title{
Role of Charge of Micellar Worms in Modulating Structure and Rheological Properties of Their Mixtures with Nonionic Polymer
}

Andrey V. Shibaev, Anton V. Makarov, Alexander I. Kuklin, Ilias Liopoulos, Olga E. Philippova

\section{To cite this version:}

Andrey V. Shibaev, Anton V. Makarov, Alexander I. Kuklin, Ilias Liopoulos, Olga E. Philippova. Role of Charge of Micellar Worms in Modulating Structure and Rheological Properties of Their Mixtures with Nonionic Polymer. Macromolecules, 2018, 51 (1), pp.213-221. 10.1021/acs.macromol.7b02246 . hal-01826372

\section{HAL Id: hal-01826372 \\ https://hal.science/hal-01826372}

Submitted on 29 Jun 2018

HAL is a multi-disciplinary open access archive for the deposit and dissemination of scientific research documents, whether they are published or not. The documents may come from teaching and research institutions in France or abroad, or from public or private research centers.
L'archive ouverte pluridisciplinaire HAL, est destinée au dépôt et à la diffusion de documents scientifiques de niveau recherche, publiés ou non, émanant des établissements d'enseignement et de recherche français ou étrangers, des laboratoires publics ou privés. 


\title{
Role of Charge of Micellar Worms in Modulating Structure and Rheological Properties of Their Mixtures with Nonionic Polymer
}

\author{
Andrey V. Shibaev, ${ }^{\dagger}$ Anton V. Makarov, ${ }^{\dagger}$ Alexander I. Kuklin, ${ }^{\ddagger}$ Ilias Iliopoulos, ${ }^{\S}$ \\ and Olga E. Philippova* ${ }^{*}+$ (i) \\ ${ }^{\dagger}$ Physics Department, Moscow State University, 119991 Moscow, Russia \\ ${ }^{\ddagger}$ Joint Institute for Nuclear Research, 141980 Dubna, Russia \\ ${ }^{\S}$ PIMM, ENSAM, CNRS, CNAM, 151 boulevard de l'Hôpital, 75013 Paris, France
}

\begin{abstract}
The paper describes phase behavior, microstructure, and rheological properties of the mixtures of surfactant micelles of varying negative charge with nonionic hydrophilic polymer poly(vinyl alcohol) (PVA) weakly interacting with the micelles. To reduce the charge of micelles, a cationic surfactant $n$-octyltrimethylammonium bromide was gradually added to the anionic surfactant potassium oleate. No appreciable interactions between the micelles and polymer were revealed by NMR spectroscopy. At the same time, the system shows a broad range of compatibility, which may be due to surfactant counterions preventing the demixing. The higher the concentration of added polymer, the higher the charge density of micelles necessary to prevent macrophase separation. However, SANS data demonstrate that upon the addition of polymer the intermicellar distances decrease, which suggests that the micelles are expelled from some area filled by PVA macromolecules,

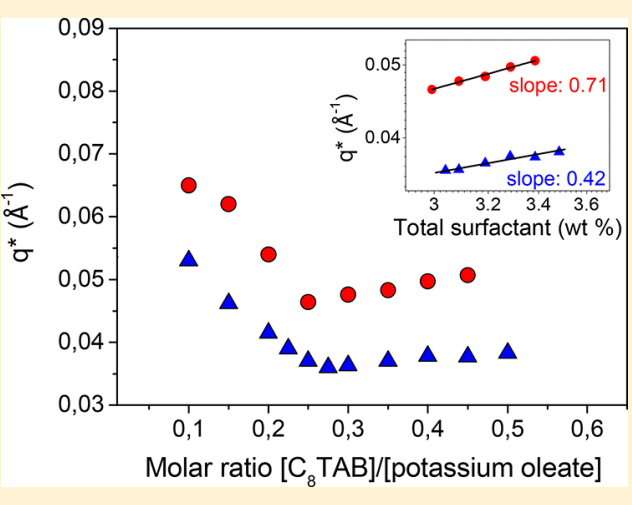
indicating a local demixing. A decrease of micellar charge density induces the growth of micelles from short nonoverlapping cylinders to long entangled wormlike chains both in the presence and in the absence of polymer, which is accompanied by an increase of viscosity by up to 5 orders of magnitude. Despite very weak interactions with the micelles, PVA makes their growth in length much more pronounced and forms the entanglements with micellar chains, thereby enhancing significantly the viscoelastic properties. The effect of polymer on micellar length was attributed to polymer-surfactant microsegregation increasing the local surfactant concentration and consequently the length of micelles. These data demonstrate the effectiveness of the addition of weakly interacting polymer for the modulation of rheology of wormlike surfactant micelles, thus opening a new way for manipulating the rheological properties of viscoelastic surfactants.
\end{abstract}

\section{INTRODUCTION}

Wormlike micelles (WLMs) of ionic surfactants can form transient networks imparting viscoelastic properties to aqueous solutions. $^{1-9}$ These properties are widely exploited for thickening and rheology-control applications in many area including cosmetics, turbulent drag reduction, oil recovery, and so on. ${ }^{1-3,5}$ A large variety of applications stimulates an appropriate modification of the viscoelastic properties of WLM solutions.

One of the ways to modify the rheology of WLMs consists in their combination with polymers. ${ }^{10-23}$ In many cases, addition of polymeric chains enhances the viscoelasticity of WLM solutions. ${ }^{10-18,23}$ In particular, an increase of viscosity by few orders of magnitude was observed at bridging the WLMs by macromolecules interacting simultaneously with different micelles. ${ }^{10-18}$ The interactions can be either electrostatic or hydrophobic, or both. The electrostatic attraction occurs when polymer is oppositely charged with respect to the micelles like in the case of cationic, cellulose-based polyelectrolyte, and anionic surfactants sodium dodecylbenzenesulfonate and sodium dodecylethoxysulfate. ${ }^{10}$ The hydrophobic interactions usually prevail when uncharged water-soluble polymers with few hydrophobic side ${ }^{11-13}$ or end ${ }^{14-16}$ groups capable to penetrate in the surfactant core are added to WLMs. Such a situation was described for uncharged hydrophobically modified (HM) polysaccharide guar with cationic surfactant erucyl bis(hydroxyethyl)methylammonium chloride (EHAC), ${ }^{11}$ for $\mathrm{HM}$ polyacrylamide with $\mathrm{EHAC}^{12}$ and cationic gemini surfactant 1,2-N, $N^{\prime}$-bis(dimethyloctadecyl)ethene ammonium bromide, ${ }^{13}$ and for poly(ethylene oxide) end-capped with alkyl groups interacting with cationic cetyltrimethylammonium tosylate $^{14,15}$ and cetylpyridinium chloride/sodium salicylate ${ }^{16}$ micelles. Hydrophobic attraction also governs the bridging of WLMs by HM polyelectrolytes of similar charge in the presence of salt as was demonstrated for partially hydrolyzed polyacrylamide mixed with anionic WLMs of potassium 
oleate. ${ }^{17}$ Both electrostatic and hydrophobic binding to the WLMs are expected for weakly charged HM polyelectrolytes carrying opposite charge with respect to the micelles. ${ }^{18}$ Independently of the nature of polymer-surfactant interactions, it is important to ensure that these interactions are not too strong to violate the geometric balance of the head and tail groups of surfactant (packing parameter) enough to destabilize the cylindrical geometry. ${ }^{19}$ Otherwise, it will lead to the disruption of WLMs as a result of cylinder-sphere transition $^{20-22}$ accompanied by the loss of viscoelastic properties or even to the precipitation of the polymersurfactant complexes. ${ }^{21}$

Recently, it was shown ${ }^{23}$ that a pronounced enhancement of rheological properties can be achieved by using a polymer weakly interacting with the micelles, if one can get a homogeneous system with sufficiently high concentration of the components providing a significant interlacement between polymer and micellar chains. This system seems to be very promising since it can be based on a variety of common commercially available water-soluble polymers. To enhance compatibility of the weakly interacting components, the micellar chains should be charged and the ionic strength of solution low, which makes demixing unfavorable, since it will induce a pronounced loss of translational entropy of surfactant counterions. $^{23}$ At the same time, the poor compatibility between polymer and micellar components leads to their microsegregation (microphase separation) with the formation of polymer-rich and surfactant-rich domains, which was evidenced by cryo-transmission electron microscopy (cryoTEM). ${ }^{23}$ The domain structure percolating the whole system gives enough space to counterions to keep high translational entropy, whereas the local segregation of poorly compatible components provides the gain in interaction energy. Charged WLMs in the absence of added salt, which are necessary for the stability of such system, can be prepared from a pair of oppositely charged surfactants ${ }^{24-28}$ (OCS). Up to now, surfactant-polymer systems were studied only for OCS micelles of constant composition, i.e., at fixed degree of charging. ${ }^{23}$ At the same time, one can suggest that the charge of micelles should play a key role in the behavior of weakly interacting surfactant-polymer system, in particular, in the compatibility of the components and in the viscoelastic properties.

Thus, the aim of this paper is to investigate the effect of the degree of charging of surfactant micelles on the rheology and the microstructure of their mixtures with nonionic polymer weakly interacting with the micelles. For this purpose, we used charged micelles of anionic surfactant potassium oleate and cationic surfactant $n$-octyltrimethylammonium bromide $\left(\mathrm{C}_{8} \mathrm{TAB}\right)$ and nonionic water-soluble polymer PVA. The charge of the OCS micelles was varied by changing the molar ratio $\beta$ of cationic and anionic surfactants. To make the effect of micellar charge clearer, no low molecular weight salt was added to the solutions. We demonstrate that lowering of the charge density of potassium oleate micelles at the addition of oppositely charged surfactant leads to the growth of micelles in length and the transition of the system from dilute to semidilute regime. The presence of polymer makes this growth much more pronounced and induces the interlacing between polymer and WLMs, which results in the significantly enhanced rheological properties. These findings provide new insights and foster a better understanding of the behavior of the mixed systems based on a combination of "living" micellar assemblies and polymeric chains, which can open the way for new practical applications of these industrially important systems.

\section{EXPERIMENTAL SECTION}

Materials. Potassium oleate (potassium cis-9-octadecanoate, $\mathrm{C}_{9} \mathrm{H}_{18}=\mathrm{C}_{8} \mathrm{H}_{15} \mathrm{COOK}$ ) was purchased from TCI (purity >98\%), $\mathrm{C}_{8} \mathrm{TAB}$ was obtained from $\mathrm{ABCR}$ (purity $>98 \%$ ), and PVA with a molar mass $27000 \mathrm{~g} / \mathrm{mol}$ (degree of polymerization $N \approx 600$ ) and residual content of acetate units 1.1-1.9\% (Mowiol 4-98) was obtained from Aldrich. All chemicals were used without further purification. Distilled deionized water (Millipore Milli-Q) was used for preparation of the samples for phase behavior and rheological studies; $\mathrm{D}_{2} \mathrm{O}$ obtained from AstraChem (Russia, $99.9 \%$ isotopic purity) was used for SANS and NMR measurements.

The critical micelle concentrations of potassium oleate and $\mathrm{C}_{8} \mathrm{TAB}$ are $\sim 0.9 \mathrm{mM}^{29}$ and $0.14 \mathrm{M}_{,}^{24}$, respectively. In all experiments, the concentration of $\mathrm{C}_{8} \mathrm{TAB}$ never surpassed $0.1 \mathrm{M}$, so this surfactant did not form micelles itself. The overlap concentration $C^{*}$ of PVA macromolecules is $0.7 \mathrm{monomol} / \mathrm{L}$ as determined by viscometric measurements. ${ }^{23}$

Sample Preparation. To obtain stock solutions, surfactants were dissolved in water overnight at room temperature. PVA stock solution was made by dissolving it in water for $1 \mathrm{~h}$ in an oil bath at $90{ }^{\circ} \mathrm{C}$. Then, the samples were prepared by mixing stock water solutions of surfactants and PVA in appropriate ratios and stirring them with a magnetic stirrer for 1-2 days. After preparation, the samples were left at room temperature for 1-3 weeks before examination.

Phase Behavior. For phase behavior studies, the samples were visually inspected 3 weeks after preparation. To determine the composition of each phase, the samples were prepared in $\mathrm{D}_{2} \mathrm{O}$ and left to phase segregate, and after that the phases were carefully separated from each other by a syringe and examined by ${ }^{1} \mathrm{H}$ NMR. For these experiments, the upper highly viscous phase was diluted $1: 1$ with $\mathrm{D}_{2} \mathrm{O}$, whereas the lower phase was studied as is.

${ }^{1} \mathrm{H}$ Nuclear Magnetic Resonance. NMR measurements were performed with an Agilent $400 \mathrm{MR}$ spectrometer operating at a frequency of $400 \mathrm{MHz}$ and equipped with an automated triple broadband detector. The samples prepared in $\mathrm{D}_{2} \mathrm{O}$ were put into standard $5 \mathrm{~mm}$ quartz tubes (Norell); the spectra were recorded at 20 ${ }^{\circ} \mathrm{C}$ and accumulated from eight scans. The data were processed using ACD Laboratories 6.0 software, including baseline and reference correction and calculation of peak integrals. Concentration ratios of different compounds in the samples were calculated from the ratio of areas under corresponding peaks.

Small-Angle Neutron Scattering. SANS measurements were done at the IBR-2 pulsed reactor, Frank Laboratory of Neutron Physics, Joint Institute for Nuclear Research (Dubna, Russia). A YuMO spectrometer was used, which has two ring detectors of scattered intensity covering the scattering vectors $q$ dynamical range 0.005-0.7 $\AA^{-1}$ due to a two-detector system. ${ }^{30,31}$ The intensity was measured in absolute units $\left(\mathrm{cm}^{-1}\right)$ by using a vanadium standard. Since most of the samples were highly viscous, they were put in the specially constructed dismountable cells with parallel quartz plates (beam path $2 \mathrm{~mm}$ ). The temperature during measurements was controlled by a water-circulating thermostat at $20.0 \pm 0.5^{\circ} \mathrm{C}$.

All compounds (surfactants and polymer) were hydrogenated; therefore, the samples were prepared in $\mathrm{D}_{2} \mathrm{O}$ to obtain high contrast. The scattering length densities of surfactant alkyl tails, polymer, and $\mathrm{D}_{2} \mathrm{O}$ are equal to $-0.30 \times 10^{-6}, 0.65 \times 10^{-6}$, and $6.36 \times 10^{-6} \AA^{-2}$, respectively. However, the scattered intensity of polymer solutions is 1-2 orders of magnitude lower than that of surfactants, since micelles are much "thicker" objects. Therefore, it was assumed that the scattered intensity is mostly contributed by surfactant micelles.

Primary treatment of the SANS data included corrections for the sample transmission, sample thickness, and electronic noise by the SAS program. ${ }^{32}$ Incoherent (background) scattering was subtracted from the data by using a blank solvent $\left(\mathrm{D}_{2} \mathrm{O}\right)$.

Fitting of the scattering curves was performed by the program SasView (http://www.sasview.org/). A part of the scattering curve at 
wave vectors $q$ higher than the structure peak position $q^{*}(0.06-0.07$ $\AA^{-1}$ ) was fitted by a form factor of an infinite cylinder (without structure factor). Two fitting parameters were used: radius of the cylinder and background level. Fitting of the scattering curves in the full $q$-range was performed by combining a finite cylinder form factor with a Hayter-Penfold potential which accounts for electrostatic repulsion of charged objects. ${ }^{33}$ The radius of the cylinder and background were fixed, and their values were taken from the high $q$ range fitting by an infinite cylinder. In the full $q$-range fit, two fitting parameters were used: length of the cylinder and its charge. In the dilute regime, a similar fitting procedure has been recently applied for elliptical charged micelles of cetyltrimethylammonium chloride with sodium salicylate, ${ }^{34}$ and in our case it allows direct calculation of the length of cylindrical micelles. In the semidilute regime, the mesh size of the entangled micellar network $\xi$ is obtained from the SANS fit instead of the micellar length, since the micelles are much longer than $\xi$, so that the largest size of an "inhomogeneity" is the mesh size, and the network is a homogeneous object on the length scales larger than $\xi^{35}$

Rheology. Rheological measurements were carried out with a stress-controlled rotational rheometer Physica MCR 301 (Anton Paar, Austria) as described elsewhere. ${ }^{36,37}$ In order to study highly viscous samples (with zero-shear viscosity $\eta_{0}>0.1 \mathrm{~Pa} \mathrm{~s}$ ), a stainless steel cone-plate (diameter $50 \mathrm{~mm}$, cone angle $1^{\circ}$ ) was used. For samples with lower viscosity $\left(\eta_{0}<0.1 \mathrm{~Pa} \mathrm{~s}\right)$, double-gap coaxial cylinders (mean diameter $26.4 \mathrm{~mm}$, height $40 \mathrm{~mm}$, gap $0.42 \mathrm{~mm}$ ) were implied. During measurements, the temperature was set at $20.00 \pm 0.05{ }^{\circ} \mathrm{C}$ by a water-circulating thermostat and Peltier elements. A special measuring cell cover filled with solvent was used to prevent solvent evaporation from the sample. To equilibrate the sample after putting it into the rheometer, it was kept inside the measuring cell for 10-30 min and subjected to low-amplitude $(0.5 \%)$ oscillatory deformation with a frequency $\omega$ of $1-10 \mathrm{~s}^{-1}$ and amplitude $0.5-3 \%$ prior to investigation. Rheological measurements were carried out at least two times to ensure that they coincide and the sample is equilibrated in the cell.

In oscillatory shear experiments, the angular frequency dependences of the storage $G^{\prime}(\omega)$ and loss $G^{\prime \prime}(\omega)$ moduli were measured. All measurements were made in the linear viscoelastic regime at the deformation amplitudes of $0.5-3 \%$, at which the storage and loss moduli were independent of deformation, as estimated previously by amplitude sweep tests performed at the frequency of $10 \mathrm{~s}^{-1}$. The plateau modulus $G_{0}$ was determined from the $G^{\prime}(\omega)$ dependence at the frequency $\omega_{\min }$ where $G^{\prime \prime}$ gets a minimum value. ${ }^{38}$ The terminal relaxation time $\tau$ was estimated as $\tau=1 / \omega_{0}$ from the frequency $\omega_{0}$ corresponding to the interception of $G^{\prime}(\omega)$ and $G^{\prime \prime}(\omega)$. In steady shear experiments, the flow curves (variation of viscosity with shear rate) were obtained. From these curves, the values of the zero-shear viscosity $\eta_{0}$ were determined by fitting the rheological data with the Carreau-Yasuda model.

\section{RESULTS AND DISCUSSION}

Phase Behavior. First, we studied the effect of charge density of surfactant micelles on the phase behavior of potassium oleate $/ \mathrm{C}_{8} \mathrm{TAB} / \mathrm{PVA}$ mixtures. In these experiments, the concentration of anionic surfactant (potassium oleate) was kept constant at $0.078 \mathrm{M}(2.5 \mathrm{wt} \%)$, whereas the concentration of cationic surfactant $\left(\mathrm{C}_{8} \mathrm{TAB}\right)$ was changed from 0.0078 to 0.1 $\mathrm{M}(0.2$ to $2.5 \mathrm{wt} \%)$, which corresponds to the variation of the molar ratio $\left[\mathrm{C}_{8} \mathrm{TAB}\right] /[$ potassium oleate $] \beta$ in the range $0.1-$ 1.3 .

The partial phase diagram of potassium oleate/ $\mathrm{C}_{8} \mathrm{TAB} / \mathrm{PVA}$ mixtures in water is presented in Figure 1. It shows that in the absence of PVA mixed surfactants form one-phase homogeneous solutions in the whole studied range of $\left[\mathrm{C}_{8} \mathrm{TAB}\right] /$ [potassium oleate] molar ratios $\beta$. In the presence of PVA, solutions are homogeneous at low molar ratios $\beta$ but phase separate when $\mathrm{C}_{8} \mathrm{TAB}$ content is increased. According to ${ }^{1} \mathrm{H}$

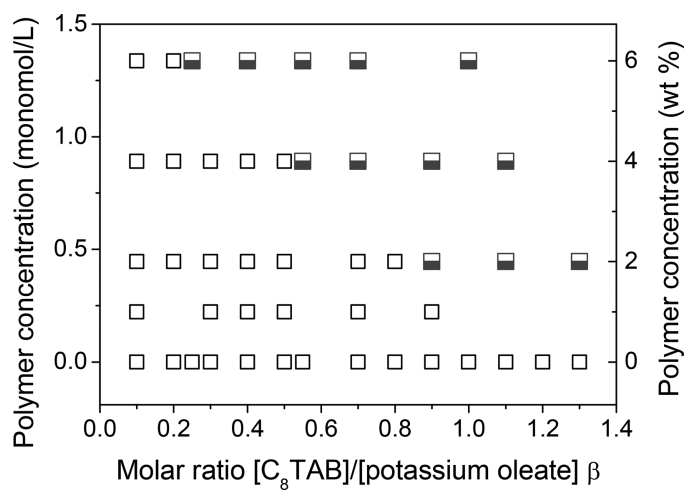

Figure 1. Phase diagram of potassium oleate $/ \mathrm{C}_{8} \mathrm{TAB} / \mathrm{PVA} /$ water system at $20{ }^{\circ} \mathrm{C}$ : open symbols, one phase; semifilled symbols, two phases. Concentration of potassium oleate: $0.078 \mathrm{M}$.

NMR spectroscopy (Figures 1S and 2S) and elemental analysis data for the demixed systems, the viscous upper phase represents the surfactant solution with some amount of polymer, whereas the lower phase of small viscosity contains only polymer, which indicates the segregation ${ }^{39}$ of the components. This is consistent with the results of ${ }^{1} \mathrm{H}$ NMR studies of model systems (potassium oleate/PVA and $\mathrm{C}_{8} \mathrm{TAB} /$ PVA) presented in Figure 3S. It is seen that the chemical shifts in the spectra of polymer-surfactants mixtures do not differ from those in the spectra of PVA (Figure 1Sb), potassium oleate (Figure $2 \mathrm{Sb}$ ) and $\mathrm{C}_{8} \mathrm{TAB}$ (Figure $2 \mathrm{Sc}$ ) taken separately. Therefore, one can conclude that the molecular environment of surfactant protons does not change upon the addition of polymer, which implies that they do not attract each other.

Similar segregative phase separation was observed, for instance, in the mixtures of two slightly incompatible polymers. ${ }^{40}$ In such systems, two counteracting factors govern the phase behavior: on the one hand, weak repulsive force between the components promotes phase separation and, on the other hand, entropic reasons countervail it. In the present system, the entropic reason counteracting demixing consists in the loss of entropy of surfactant counterions, when they accumulate mainly in one phase.

At low $\mathrm{C}_{8} \mathrm{TAB}$ content, the micelles are strongly negatively charged, and the phase separation is unfavorable for entropic reasons. When the molar ratio $\left[\mathrm{C}_{8} \mathrm{TAB}\right] /[$ potassium oleate $] \beta$ becomes higher, the net charge of micelles decreases alongside with the amount of their counterions, and above a certain point the entropy losses become less important than the repulsion between the micellar and polymer chains, and the phase separation occurs. Note that the WLMs are "living" objects, and the decrease of their charge density is inevitably accompanied by their growth in length. ${ }^{4,41}$ Indeed, with decreasing charge density the electrostatic repulsion in the corona of micelles becomes smaller, which makes tighter packing of surfactant molecules in the middle cylindrical part of the micelle more favorable in comparison with the semispherical ends, thus reducing the fraction of the end-caps in the system A smaller fraction of end-caps at the same amount of surfactant suggests longer micelles. Increased length of micelles can also contribute to the demixing, since longer chains are poorer in entropy, but this entropic contribution is much smaller than the entropy of counterions.

From Figure 1 it is seen that the region of phase compatibility narrows with the increase of polymer concentration; i.e., the higher the amount of added PVA, the more 
charged micelles can be demixed into a separate phase. This is due to the enhancement of the repulsion between the components. At the largest studied polymer concentration (1.34 monomol/L), the solutions are homogeneous only for highly charged (and short) micelles at very low molar ratios $\beta$ $\leq 0.2$. Further experiments were performed only with homogeneous samples.

Structure. To investigate the structural changes, which occur in the potassium oleate/ $\mathrm{C}_{8} \mathrm{TAB} / \mathrm{PVA}$ system at varying micellar charge, SANS was used. In the experiments, the concentration of PVA was kept at 0.89 monomol/L (4 wt \%), which is slightly higher than its overlap concentration in water $\left(C^{*}=0.7 \mathrm{monomol} / \mathrm{L}\right){ }^{23}$ The concentration of potassium oleate was equal to $0.078 \mathrm{M}$, whereas the value of the molar ratio $\left[\mathrm{C}_{8} \mathrm{TAB}\right] /[$ potassium oleate $] \beta$ was varied from 0.1 to 0.45 , which corresponds to homogeneous solutions (Figure 1) with strongly negatively charged micelles. Since surfactant micelles in $\mathrm{D}_{2} \mathrm{O}$ scatter neutrons much stronger than PVA (Figure 4S), the SANS curves in mixed surfactant/polymer systems represent mainly the scattering from the micellar component.

Effect of Micellar Charge. Figure 2a shows the evolution of the scattering pattern with decreasing degree of charge of micelles (i.e., increasing amount of added $\mathrm{C}_{8} \mathrm{TAB}$ ) at fixed concentrations of both potassium oleate and PVA. It is seen that in the high $q$-range (at $q>0.07 \AA^{-1}$ ) the curves superimpose perfectly, indicating that the local structure of
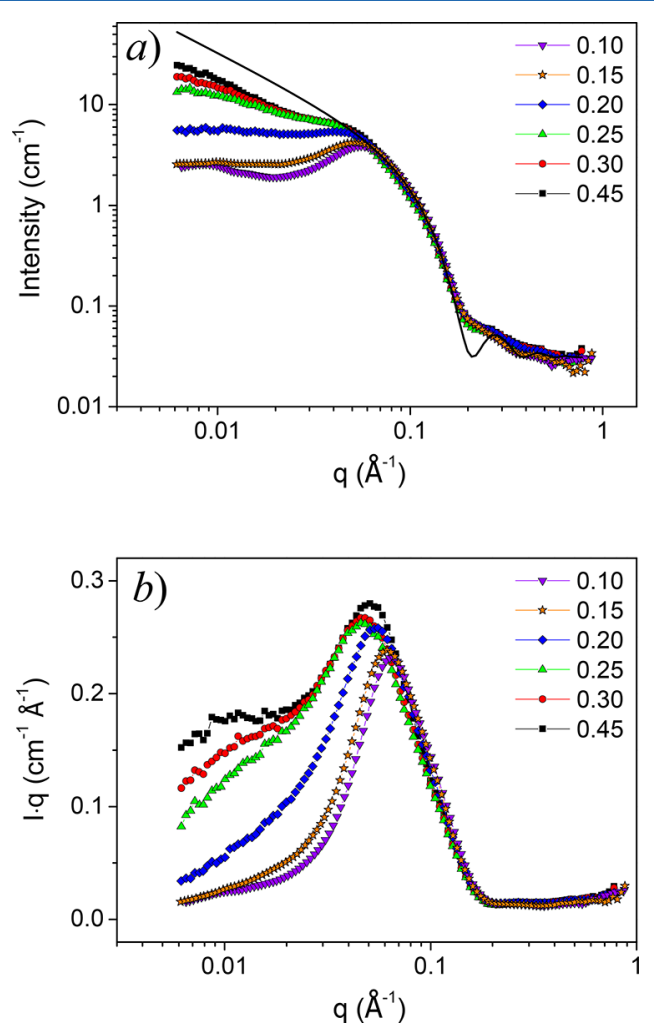

Figure 2. Scattering curves in $I$ versus $q$ (a) and $I \cdot q$ versus $q$ (b) representations for $0.078 \mathrm{M}$ aqueous solutions of potassium oleate containing 0.89 monomol/L PVA and different amounts of $\mathrm{C}_{8} \mathrm{TAB}$ corresponding to molar ratios $\left[\mathrm{C}_{8} \mathrm{TAB}\right] /[$ potassium oleate $] \beta: 0.10$ (violet reverse triangles), 0.15 (red stars), 0.20 (blue diamonds), 0.25 (green triangles), 0.30 (red circles), 0.45 (black squares) at $20{ }^{\circ} \mathrm{C}$. Solid line is a fit of the scattering curves by a form factor of a cylinder with radius $R=18.1 \pm 0.4 \AA$. surfactant aggregates is the same independently of the micellar charge. In this range, the curves can be fitted by a form factor of a cylinder with the radius of $18.1 \pm 0.4 \AA$ (Figure $2 \mathrm{a}$ ), which is close to the length of oleate tail $(19 \AA)^{42}$ and is much larger than the length of $\mathrm{C}_{8} \mathrm{TAB}$ alkyl group $(8 \AA)$, suggesting that at the studied molar ratios $\beta=0.1-0.45$ the radius of an OCS micelle is governed by the size of the longer anionic surfactant tail and does not depend on the content of $\mathrm{C}_{8} \mathrm{TAB}$. Similar behavior was previously observed for sodium oleate $/ \mathrm{C}_{8} \mathrm{TAB}$ micelles in the absence of polymer. ${ }^{24}$ Fitting of the high $q$-range of the scattering curves in order to obtain the micellar radii was applied for several charged surfactant-micellar systems. ${ }^{24,43}$ Thus, according to SANS data, at $\beta$ values under study, the micelles of locally cylindrical shape are always present in potassium oleate $/ \mathrm{C}_{8} \mathrm{TAB} / \mathrm{PVA}$ solution, and their radius is not altered by the amount of added $\mathrm{C}_{8} \mathrm{TAB}$.

At the same time, at low $q$-values $\left(q<0.05 \AA^{-1}\right)$ reflecting larger scale structural features the scattering curve is strongly affected by the charge of micelles: with decreasing charge density (i.e., with increasing molar ratio $\beta$ ) the scattered intensity $I$ is greatly enhanced by nearly an order of magnitude (see also the scattering curves in linear representation on Figure 5S). An increase in SANS intensity at low $q$ was previously observed at the screening of the micellar charges with low molecular weight salt ${ }^{43}$ or oppositely charged surfactant. ${ }^{24}$ It was attributed to the elongation of cylindrical micelles. Indeed, addition of oppositely charged surfactant produces two main effects: (i) the decrease of the net charge of micelles and (ii) the release of counterions increasing the ionic strength of the solution. ${ }^{24}$ Both these effects reduce the electrostatic repulsion in the corona of the micelles, thus favoring tighter packing of surfactant molecules in a cylinder at the expense of spherical end-caps. The lower the fraction of end-caps, the longer the micelles. In our work, we show that growth of cylindrical micelles in length with decreasing their charge density occurs also in the presence of PVA. Besides, one should note that decreasing charge of micelles will make the intermicellar repulsive interactions weaker, thereby reducing the contribution of the structure factor. As the structure factor decreases the scattering intensity at low $q$, the lowering of its contribution will enhance the intensity. So, the increase of scattering intensity at low $q$ can be attributed to two reasons: elongation of micelles and reducing of the intermicellar repulsion. One can expect that the first reason will be important mainly in dilute solutions because in semidilute regime the scattering will characterize the mesh size of the transient network.

Figure 2a demonstrates that at low $q$ the intensity $I$ of experimental curves is smaller than the predicted cylinder form factor, which can be assigned to strong electrostatic repulsion between the similarly charged cylindrical micelles, ${ }^{23,44}$ that is, to the contribution of the structure factor. The scattering curves of surfactant micelles (in the absence of polymer) can be wellfitted in the whole $q$-range by a model combining a finite cylinder form factor with a Hayter-Penfold potential ${ }^{33}$ which accounts for electrostatic repulsion of charged objects (Figure $6 \mathrm{~S})$. The fitting gives an estimate of the length of micelles (Table 1S): with increasing $\beta$ from 0.2 to 0.275 (dilute regime) the length of micelles grows from 28 to $40 \mathrm{~nm}$. Note that in this part of the curve we do not observe the scaling dependence $I \sim$ $q^{-1}$ typical for cylindrical objects ${ }^{42}$ at $q R \ll 1$ because their form factor is hidden by the contribution of the structure factor. $^{24}$ 
At intermediate $q$-values $\left(q=0.05-0.07 \AA^{-1}\right)$ a correlation peak is observed. It is better seen in $I \cdot q$ versus $q$ representation (Figure 2b) which eliminates the contribution of the form factor in this part of the curve. ${ }^{45}$ The structure peak characterizes the mutual arrangement of similarly charged micelles resulting from electrostatic repulsion between them, which should be rather strong since no salt was added to shield the repulsion. The variation of the position of the peak $q^{*}$ with molar ratio $\left[\mathrm{C}_{8} \mathrm{TAB}\right] /[$ potassium oleate $] \beta$ for the potassium oleate $/ \mathrm{C}_{8} \mathrm{TAB} / \mathrm{PVA}$ system is illustrated in Figure 3 (red

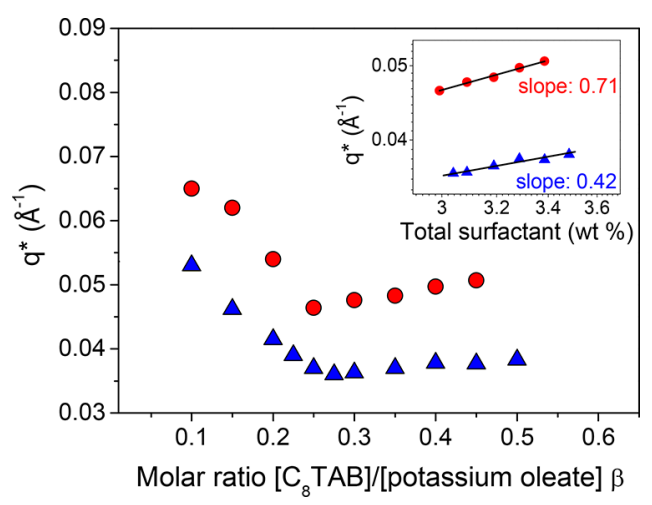

Figure 3. Dependences of the position of the correlation peak $q^{*}$ on the molar ratio $\left[\mathrm{C}_{8} \mathrm{TAB}\right] /[$ potassium oleate $] \beta$ and on the total surfactant concentration (inset) for $0.078 \mathrm{M}$ aqueous solutions of potassium oleate with different concentrations of $\mathrm{C}_{8} \mathrm{TAB}$ in the absence (blue triangles) and in the presence of 0.89 monomol/L PVA (red circles) at $20^{\circ} \mathrm{C}$. The straight lines are power law fits yielding the exponents of 0.42 and 0.71 in the absence and in the presence of polymer, respectively.

circles). It is seen that the curve consists of two distinct regions. In the first one $(0.1<\beta<0.25)$, the lowering of the micellar charge induces a pronounced decrease of $q^{*}$, indicating that the intermicellar distances $2 \pi / q^{*}$ become much longer, which can be due to the growth of micelles in length. ${ }^{46}$ Indeed, from simple geometrical considerations it can be concluded that in the dilute regime the average distance between longer cylindrical objects should be larger than between the shorter ones made of the same amount of material (the concentration of $\mathrm{C}_{8} \mathrm{TAB}$ added in this range is several times lower than that of potassium oleate). At the molar ratio $\beta \approx 0.25$, a sharp inflection is seen at the curve (Figure 3). A similar dependence with an inflection point was observed in the literature for cylindrical micelles of cetyltrimethylammonium tosylate at increasing concentration of surfactant, and the inflection was attributed to the crossover from dilute to semidilute regime. ${ }^{46}$ Although in our system the composition of the surfactant mixture $\beta$ (instead of the concentration of surfactant) was varied, one can suggest that at $\beta \approx 0.25$ the transition to semidilute regime occurs. Thus, the first region on the curve $(0.1<\beta<0.25)$ corresponds to the dilute regime, whereas the second one $(\beta>0.25)$ to semidilute regime (Figure 3$)$. In semidilute regime, the micelles overlap and their growth soon results in the formation of an entangled network (as will be evidenced later by the rheological data). In the semidilute entangled regime, $2 \pi / q^{*}$ roughly corresponds to the mesh size $\xi$ of the network. ${ }^{46}$ As seen from Figure 3, in the second region $(\beta>0.25), q^{*}$ slightly increases with $\mathrm{C}_{8} \mathrm{TAB}$ concentration, indicating the reduction of $\xi$, i.e., the formation of denser network. Plotting $q^{*}$ vs total (potassium oleate and $\mathrm{C}_{8} \mathrm{TAB}$ ) surfactant concentration (Figure 3, inset) gives a straight line. The slope of this line is close to the theoretical dependence ${ }^{46}$ for strongly interacting cylindrical objects in semidilute regime: $q^{*} \sim C^{0.5}$. Therefore, the observed slight growth of $q^{*}$ in the second region can be simply explained by the increase of the total amount of surfactant molecules building the micelles.

Thus, the analysis of the SANS data suggests that decreasing micellar charge induces the growth of cylindrical micelles in length in the presence of polymer, which leads finally to the transition from dilute to semidilute regime at $\beta \approx 0.25$ and to the formation of a polymer-micellar network.

Effect of Polymer. In order to reveal the role of polymer in the microstructure formation, let us compare the scattering curves of potassium oleate $/ \mathrm{C}_{8} \mathrm{TAB}$ solutions in the presence and in the absence of PVA at a constant molar ratio $\left[\mathrm{C}_{8} \mathrm{TAB}\right] /$ [potassium oleate] $\beta$. As depicted in Figure 4, at intermediate $q$ -

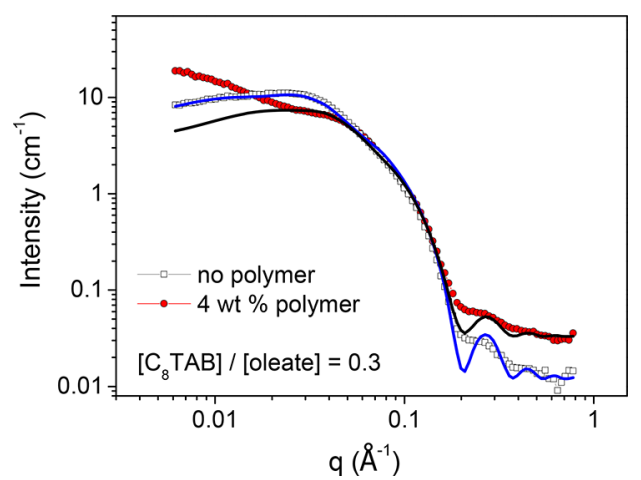

Figure 4. Scattering curves for aqueous solutions containing $0.078 \mathrm{M}$ potassium oleate and $0.023 \mathrm{M} \mathrm{C}_{8} \mathrm{TAB}$ (molar ratio [ $\left.\mathrm{C}_{8} \mathrm{TAB}\right]$ ) [potassium oleate] $\beta=0.3$ ) in the absence (open symbols) and in the presence of 0.89 monomol/L PVA (filled symbols) at $20^{\circ} \mathrm{C}$. Solid lines are fits of the scattering curves by a model of finite cylinder with electrostatic repulsion with the Hayter-Penfold potential.

values $\left(0.06 \AA^{-1}<q<0.2 \AA^{-1}\right)$, the curves coincide with each other showing that the local structure of surfactant aggregates is not affected by PVA. A clear effect of polymer is seen at high and at low $q$-ranges (Figure 4$)$. At high $q$-range $\left(q>0.2 \AA^{-1}\right)$, polymer increases the background intensity of the scattering curve (cf. Figure $4 S$ ). In the low $q$-range, the effect of polymer is more complicated. Without PVA, the scattered intensity levels off at small $q$. This may be explained by the formation of an entangled micellar network (which will be evidenced later by the rheological data). The network can be regarded as a "homogeneous" object at length scales larger than its mesh size (i.e., there are no larger inhomogeneities than one mesh), which results in the constant intensity at low $q$-range. ${ }^{35}$ At the same time, in the presence of PVA, the intensity is higher, and moreover it grows with decreasing $q$, which indicates the formation of structures larger than $\sim 1 / q_{\min }=150 \mathrm{~nm}$. This is consistent with our recent cryo-TEM data ${ }^{23}$ on potassium oleate $/ \mathrm{C}_{8} \mathrm{TAB} / \mathrm{PVA}$ mixtures demonstrating the formation of large (several hundreds of nanometers) domains enriched either by WLMs or by polymer, which arise from poor compatibility between polymer and surfactant micelles. These domains seem to be responsible for the enhancement of intensity at low $q$. Therefore, if we return to the scattering curves at different molar ratios $\beta$ (Figure 2 and Figure 5S), we can state that in the presence of PVA the rise of low- $q$ intensity at decreasing charge density of micelles proceeds due to three 
reasons (formation of microdomains, increase of micellar length, and reduction of repulsive interactions), whereas in pure surfactant only last two reasons (the growth of micelles ${ }^{24}$ and the decrease of repulsion) will contribute to this effect. Indeed, as was discussed above, the scattering curve of the surfactant micelles (without PVA) is well-fitted in the whole $q$-range by a model combining a finite cylinder form factor with the HayterPenfold potential $^{33}$ which accounts for electrostatic repulsion of charged objects (Figure 4 and Figure 6S). Similar fitting of polymer-surfactant system deviates from the experimental scattering curve (Figure 4) at low $q$-values, indicating additional factor affecting the scattering, which most probably concerns the polymer-surfactant microsegregation with the formation of microdomain structure.

Figure 3 demonstrates the influence of polymer on the dependence of the structure peak position $q^{*}$ on the molar ratio $\left[\mathrm{C}_{8} \mathrm{TAB}\right] /[$ potassium oleate $] \beta$. It is seen that the curves with and without PVA show similar behavior, suggesting that the general structural evolution of micelles with the decrease of their charge proceeds in the same way in both cases. The most significant effect produced by polymer consists in the shift of the whole curve to higher $q^{*}$-values, indicating that at all micellar charges under study (i.e., at all $\left[\mathrm{C}_{8} \mathrm{TAB}\right] /[$ potassium oleate] molar ratios $\beta$ ) the average distance between the micelles decreases when PVA is added. This implies the local concentrating of micelles induced by their segregation from polymeric chains poorly compatible with the micelles. Indeed, as the surfactant micelles cannot occupy some area filled by PVA macromolecules, they need to come closer to each other upon addition of polymer; i.e., their local concentration increases. This should lead to the additional elongation of micellar chains, since the length of wormlike micelles grows with their concentration. ${ }^{4,41}$ Also, Figure 3 shows that in the presence of PVA the minimum on the curve $q^{*}(\beta)$ is slightly shifted to lower $\beta$, indicating that the overlap concentration $C^{*}$ of the micelles in polymer-surfactant system is observed for more charged micelles, which may be due to their additional elongation caused by polymer.

Thus, SANS data suggest that in the presence of polymer the local concentration of micelles and their length are higher in comparison with PVA-free micellar solutions and that the inhomogeneities at the scale larger than ca. $150 \mathrm{~nm}$ are formed. This is consistent with the behavior described by us recently ${ }^{23}$ for potassium oleate/C8TAB/PVA solutions at fixed $\beta=0.4$ and can be assigned to the local segregation of polymer and surfactant components in the solution.

Rheology. In order to relate the structural changes in potassium oleate $/ \mathrm{C}_{8} \mathrm{TAB} / \mathrm{PVA}$ solutions with their macroscopic behavior, rheological studies were performed. Figures 5 and 6 show the flow curves and the dynamic rheological data at different $\left[\mathrm{C}_{8} \mathrm{TAB}\right] /[$ potassium oleate $]$ molar ratios $\beta$ and fixed concentration of PVA $(0.89 \mathrm{monomol} / \mathrm{L})$, whereas Figure 7 depicts the variation of zero-shear viscosity $\eta_{0}$, relaxation time $\tau$, and plateau modulus $G_{0}$ with increasing $\beta$ at different amounts of polymer. In all the experiments the concentration of potassium oleate was equal to $0.078 \mathrm{M}$.

Effect of Micellar Charge. Let us first consider the evolution of rheological data with decreasing micellar charge (i.e., at increasing $\beta$ ) at constant PVA concentration of 0.89 monomol/ L. The corresponding viscosity results are presented on Figures 5 and $7 \mathrm{a}$ (red curve). From Figure $7 \mathrm{a}$, it is seen that at $\beta=0.1$ the viscosity is low $(0.006 \mathrm{~Pa} \mathrm{~s})$ and only slightly higher than the viscosity of $0.89 \mathrm{monomol} / \mathrm{L}$ PVA solution without

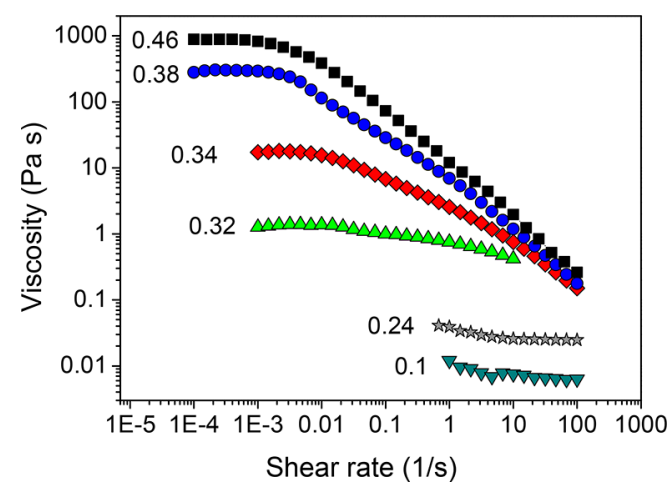

Figure 5. Flow curves of $0.078 \mathrm{M}$ potassium oleate aqueous solutions containing 0.89 monomol/L PVA and different amounts of $\mathrm{C}_{8} \mathrm{TAB}$ corresponding to molar ratios $\left[\mathrm{C}_{8} \mathrm{TAB}\right] /[$ potassium oleate $] \beta: 0.1$ (reverse cyan triangles), 0.24 (gray stars), 0.32 (green triangles), 0.34 (red diamonds), 0.38 (blue circles), and 0.46 (black squares) at $20{ }^{\circ} \mathrm{C}$.

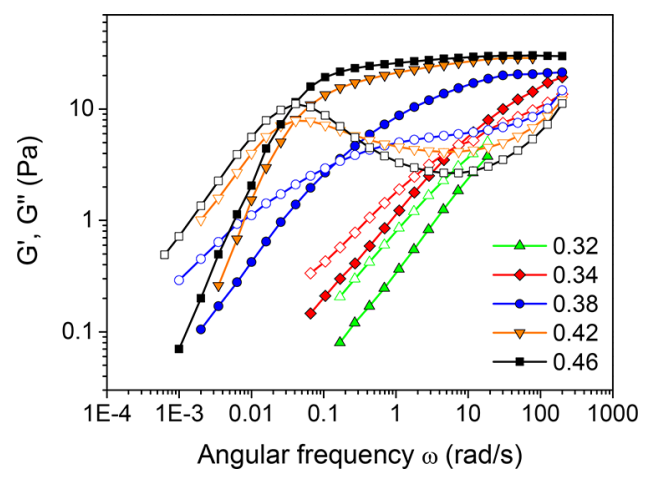

Figure 6. Frequency dependences of storage $G^{\prime}$ (filled symbols) and loss $G^{\prime \prime}$ (open symbols) moduli for $0.078 \mathrm{M}$ potassium oleate aqueous solutions containing 0.89 monomol/L PVA and different amounts of $\mathrm{C}_{8} \mathrm{TAB}$ corresponding to molar ratios $\left[\mathrm{C}_{8} \mathrm{TAB}\right] /[$ potassium oleate $] \beta$ : 0.32 (green triangles), 0.34 (red diamonds), 0.38 (blue circles), 0.42 (orange reverse triangles), and 0.46 (black squares) at $20{ }^{\circ} \mathrm{C}$.

surfactants (0.005 $\mathrm{Pa} \mathrm{s})$, which is in accordance with the presence of rather short cylindrical micelles. When the molar ratio $\beta$ is raised from 0.1 to 0.23 , the viscosity becomes only slightly higher suggesting the elongation of cylindrical micelles in dilute regime. At molar ratio $\beta \approx 0.23$, the rheological behavior changes drastically. The zero-shear viscosity starts to rise sharply (Figure 7a), indicating the transition from dilute to semidilute regime, and finally grows by nearly 4 orders of magnitude, reaching the value of $900 \mathrm{~Pa}$ s at $\beta=0.46$. As seen from flow curves (Figure 5), viscous solutions exhibit shearthinning behavior.

Dynamic rheological data (Figure 6) demonstrate that with decreasing micellar charge at the addition of $\mathrm{C}_{8} \mathrm{TAB}$ the solutions progressively gain viscoelastic properties. The intercept point between $G^{\prime}(\omega)$ and $G^{\prime \prime}(\omega)$ dependences shifts to lower frequency indicating the increase of the relaxation time $\tau$. Note that the present systems do not show simple Maxwell behavior with a single relaxation time; ${ }^{23}$ therefore, the relaxation time determined from the intersection of $G^{\prime}(\omega)$ and $G^{\prime \prime}(\omega)$ curves can be regarded as a terminal one. At molar ratios $\beta>0.38$, one can observe a well-defined high-frequency plateau on $G^{\prime}(\omega)$ dependence indicating to the formation of an entangled wormlike micellar network. ${ }^{4,6,47}$ The dynamic rheological data for lower polymer concentration 0.22 monomol/L are quite similar (Figure $7 \mathrm{~S}$ ). Overall, the observed 

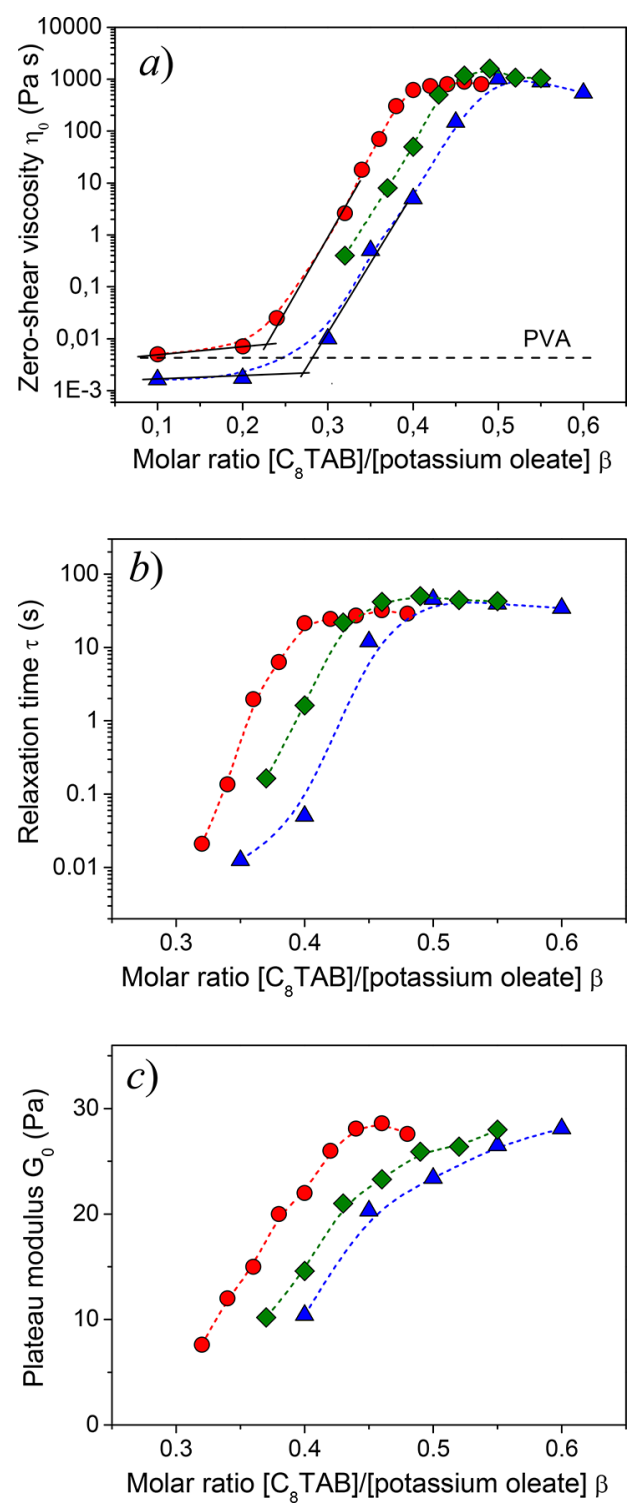

Figure 7. Dependences of zero-shear viscosity $\eta_{0}$ (a), terminal relaxation time $\tau(\mathrm{b})$, and plateau storage modulus $G_{0}(\mathrm{c})$ on the molar ratio $\left[\mathrm{C}_{8} \mathrm{TAB}\right] /[$ potassium oleate $] \beta$ for $0.078 \mathrm{M}$ aqueous solutions of potassium oleate containing $\mathrm{C}_{8} \mathrm{TAB}$ and different concentrations of PVA: 0 (blue triangles), $0.22 \mathrm{monomol} / \mathrm{L}$ (green diamonds), and 0.89 monomol/L (red circles). Dashed black line (a) indicates the zeroshear viscosity of 0.89 monomol/L PVA solution.

evolution of rheological properties can be explained by the elongation of micelles upon decreasing their charge leading to the transition of the solution to semidilute regime.

Figures $7 \mathrm{a}$ and $7 \mathrm{~b}$ show that $\eta_{0}$ and $\tau$ grow up to maximum and slightly decrease after it. The maximum on the viscosity curves versus salt concentration is usually attributed to the formation of branching points on WLMs. ${ }^{48-51}$ Unlike branches in polymers, micellar branches are labile. They can slide along a micelle contour, thus accelerating the stress relaxation and therefore decreasing relaxation time $\tau$ and the zero-shear viscosity $\eta_{0}$. Although this mechanism of the viscosity reduction is widely accepted, a thorough study of sodium oleate $/ \mathrm{C}_{8} \mathrm{TAB}$ micelles (in the absence of polymer) by cryo-TEM ${ }^{25}$ has evidenced that the maximum of viscosity as a function of the composition of the surfactant mixture is not related to the branching, but it is due to the formation of longest linear
WLMs followed by a shortening of worms at higher amount of added $\mathrm{C}_{8} \mathrm{TAB}$. The same seems to be true for the system with PVA. As for plateau modulus values $G_{0}$ (Figure $7 \mathrm{c}$ ), they continue to get higher when zero-shear viscosity $\eta_{0}$ and terminal relaxation time $\tau$ already stay constant or even slightly decrease. As was shown for polymer-free sodium oleate $/ C_{8} T A B$ system, ${ }^{24}$ in the vicinity of the viscosity maximum the value of $G_{0}$ is independent of the composition of OCS micelles; therefore, one can suggest that the rise of $G_{0}$ with $\beta$ can be due to the increase of the total concentration of surfactants upon addition of $\mathrm{C}_{8} \mathrm{TAB}$.

Effect of Polymer. Let us reveal the role of polymer in the evolution of rheological properties with decreasing charge density of micelles by comparing the data at different content of PVA and in the absence of PVA (Figure 7). First, one can note that in the presence of polymer the transition to semidilute regime corresponding to the onset of sharp rise of viscosity (Figure 7a) proceeds at lower $\beta$ ( 0.23 instead of 0.27 for polymer-free system), which fully agrees with SANS data (Figure 3). As was discussed above, this is due to longer micelles observed in polymer-surfactant system. Second, in the presence of polymer the formation of the network of entanglements reflected in the appearance of the plateau modulus (Figure 6 and Figure 8S) also occurs at smaller molar ratio $\beta$ (0.38 instead of 0.41 for polymer-free surfactants), that is, for more charged micelles. Therefore, polymer decreases the $\beta$ values corresponding to the transition from the unentangled to entangled semidilute regime. Third, one can state that at any $\beta$ value before the maximum polymer increases significantly the viscosity of the system (Figure 7a). For example, at $\beta=0.4$ the zero-shear viscosity of polymer-surfactant system containing 0.89 monomol/L PVA is $617 \mathrm{~Pa} \mathrm{~s}$, whereas for surfactant solution it equals only $5 \mathrm{~Pa}$ s and for PVA solution $0.005 \mathrm{~Pa}$. Therefore, the viscosity of the mixed polymer-surfactant system is by more than 2 orders of magnitude higher than the viscosity of its components taken separately. Such a pronounced synergistic effect can be attributed not only to higher length of micelles in the presence of PVA. As was shown recently, ${ }^{23}$ the entanglements between polymer and micellar chains can also contribute to enhanced viscosity. Note that the concentration of PVA in the system is near the overlap concentration $C^{*}(0.7$ monomol/L $)$ and well below the entanglement concentration $C_{\mathrm{e}}$, which is usually 5-10 times higher than $C^{*}{ }^{52}$ Therefore, the polymer-polymer entanglements can be neglected. Thus, an enhancement of viscosity in semidilute regime in the presence of polymer seems to be due to the elongation of micelles induced by PVA and to the formation of the entanglements between micellar and polymeric chains. For the same reason the polymer increases the terminal relaxation time and the plateau modulus (Figure 7 ) as well as the $\beta$ values corresponding to the transition from the unentangled to entangled semidilute regime. At the same time, the maximum values of viscosity and terminal relaxation time do not depend on the presence of PVA (Figure 7). Therefore, PVA helps to reach the maximum viscosity and relaxation time at smaller $\beta$, i.e., at higher degree of charging. Thus, the addition of nonionic polymer instead of an ionic compound (for instance, cationic surfactant) may help to obtain a solution of highly charged micelles with high rheological properties, whereas in the absence of polymer the micelles need to be less charged in order to have the same viscosity and elasticity. 


\section{CONCLUSIONS}

In this paper, we studied the behavior of the solutions of ionic surfactant micelles of variable charge and nonionic polymer PVA. To decrease the degree of charging of micelles, we gradually added a cationic surfactant $\mathrm{C}_{8} \mathrm{TAB}$ to oppositely charged potassium oleate micelles. We used a combination of rheological measurements exploring the macroscopic properties (viscosity, elasticity, etc.) of the system and SANS revealing the structural rearrangements occurring at the microscopic level. We show that decreasing charge of micelles promotes their elongation and consequently the enhancement of the rheological properties (zero-shear viscosity, terminal relaxation time, and plateau modulus) both in the absence and in the presence of polymer. At a given degree of charging of micelles, the polymer induces the formation of longer micellar chains and of polymer-micellar entanglements and therefore increases significantly the rheological characteristics. We explain the role of polymer in the elongation of micelles by its local segregation from surfactant, which reduces the volume of solution accessible for the micelles, thus increasing their local concentration and promoting their growth in length. This suggestion is confirmed by SANS data, which demonstrate (i) a shift of the structure peak position toward high $q$-values upon addition of polymer indicating the decrease of intermicellar distance and (ii) an enhancement of low- $q$ scattering pointing to the formation of longer micelles and large-scale inhomogeneities.

The findings reported here demonstrate the effectiveness of the addition of weakly interacting polymer for the modulation of rheology of wormlike surfactant micelles, thus opening a new way for manipulating the rheological properties of viscoelastic surfactants.

\section{ASSOCIATED CONTENT}

\section{S Supporting Information}

The Supporting Information is available free of charge on the ACS Publications website at DOI: 10.1021/acs.macromol.7b02246.

$$
\text { NMR spectroscopy, SANS, and rheology data (PDF) }
$$

\section{AUTHOR INFORMATION}

\section{Corresponding Author}

*(O.E.P.) Fax +7 495 9392988; Tel +7 495 9391464; e-mail phil@polly.phys.msu.ru.

\section{ORCID}

Olga E. Philippova: 0000-0002-1098-0255

Notes

The authors declare no competing financial interest.

\section{ACKNOWLEDGMENTS}

This work was supported by the Russian Science Foundation (project no. 15-13-00114). The authors express their gratitude Ms. T. A. Ganina for her help in NMR measurements and to Mrs. A. L. Aleshina for the preparation of some samples for NMR experiments.

\section{REFERENCES}

(1) Zana, R., Kaler, E. W., Eds.; Giant Micelles: Properties and Applications; CRC Press: Boca Raton, FL, 2007.

(2) Feng, Y.; Chu, Z.; Dreiss, C. A. Smart Wormlike Micelles: Design, Characteristics and Applications; Springer-Verlag: Berlin, 2015.
(3) Dreiss, C. A., Feng, Y., Eds.; Wormlike Micelles: Advances in Systems, Characterisation and Applications; Royal Society of Chemistry: London, 2017.

(4) Magid, L. J. The Surfactant-Polyelectrolyte Analogy. J. Phys. Chem. B 1998, 102, 4064-4074.

(5) Yang, J. Viscoelastic Wormlike Micelles and their Applications. Curr. Opin. Colloid Interface Sci. 2002, 7, 276-281.

(6) Dreiss, C. A. Wormlike Micelles: where Do we Stand? Recent Developments, Linear Rheology and Scattering Techniques. Soft Matter 2007, 3, 956-970.

(7) Chu, Z.; Dreiss, C. A.; Feng, Y. Smart Wormlike Micelles. Chem. Soc. Rev. 2013, 42, 7174-7203.

(8) Zhao, M.; Gao, M.; Dai, C.; Zou, C.; Yang, Z.; Wu, X.; Liu, Y.; Wu, Y.; Fang, S.; Lv, W. Investigation of Novel Triple-Responsive Wormlike Micelles. Langmuir 2017, 33, 4319-4327.

(9) Morishima, K.; Sugawara, S.; Yoshimura, T.; Shibayama, M. Structure and Rheology of Wormlike Micelles Formed by Fluorocarbon-Hydrocarbon-Type Hybrid Gemini Surfactant in Aqueous Solution. Langmuir 2017, 33, 6084-6091.

(10) Hoffmann, I.; Heunemann, P.; Prevost, S.; Schweins, R.; Wagner, N. J.; Gradzielski, M. Self-Aggregation of Mixtures of Oppositely Charged Polyelectrolytes and Surfactants Studied by Rheology, Dynamic Light Scattering and Small-Angle Neutron Scattering. Langmuir 2011, 27, 4386-4396.

(11) Couillet, I.; Hughes, T.; Maitland, G.; Candau, F. Synergistic Effects in Aqueous Solutions of Mixed Wormlike Micelles and Hydrophobically Modified Polymers. Macromolecules 2005, 38, 52715282.

(12) Shashkina, J. A.; Philippova, O. E.; Zaroslov, Y. D.; Khokhlov, A. R; Pryakhina, T. A.; Blagodatskikh, I. V. Rheology of Viscoelastic Solutions of Cationic Surfactant. Effect of Added Associating Polymer. Langmuir 2005, 21, 1524-1530.

(13) Chen, F.; Wu, Y.; Wang, M.; Zha, R. Self-assembly Networks of Wormlike Micelles and Hydrophobically Modified Polyacrylamide with High Performance in Fracturing Fluid Application. Colloid Polym. Sci. 2015, 293, 687-697.

(14) Yoshida, T.; Taribagil, R.; Hillmyer, M. A.; Lodge, T. P. Viscoelastic Synergy in Aqueous Mixtures of Wormlike Micelles and Model Amphiphilic Triblock Copolymers. Macromolecules 2007, 40, $1615-1623$

(15) Lodge, T. P.; Taribagil, R.; Yoshida, T.; Hillmyer, M. A. SANS Evidence for the Cross Linking of Wormlike Micelles by a Model Hydrophobically Modified Polymer. Macromolecules 2007, 40, 47284731.

(16) Ramos, L.; Ligoure, C. Structure of a New Type of Transient Network: Entangled Wormlike Micelles Bridged by Telechelic Polymers. Macromolecules 2007, 40, 1248-1251.

(17) Molchanov, V. S.; Philippova, O. E. Dominant Role of Wormlike Micelles in Temperature-Responsive Viscoelastic Properties of their Mixtures with Polymeric Chains. J. Colloid Interface Sci. 2013, 394, 353-359.

(18) Peiffer, D. G. Hydrophobically Associating Polymers and their Interactions with Rod-like Micelles. Polymer 1990, 31, 2353-2360.

(19) Oikonomou, E.; Bokias, G.; Kallitsis, J. K.; Iliopoulos, I. Formation of Hybrid Wormlike Micelles upon Mixing Cetyl Trimethylammonium Bromide with Poly(methyl methacrylate-cosodium styrene sulfonate) Copolymers in Aqueous Solution. Langmuir 2011, 27, 5054-5061.

(20) Brackman, J. C.; Engberts, J. B. F. N. Influence of Polymers on the Micellization of Cetyltrimethylammonium Salts. Langmuir 1991, 7, 2097-2102.

(21) Li, X.; Lin, Z.; Cai, J.; Scriven, L. E.; Davis, H. T. PolymerInduced Microstructural Transitions in Surfactant Solutions. J. Phys. Chem. 1995, 99, 10865-10878.

(22) Lin, Z.; Eads, C. D. Polymer-Induced Structural Transitions in Oleate Solutions: Microscopy, Rheology, and Nuclear Magnetic Resonance Studies. Langmuir 1997, 13, 2647-2654.

(23) Shibaev, A. V.; Abrashitova, K. A.; Kuklin, A. I.; Orekhov, A. S.; Vasiliev, A. L.; Iliopoulos, I.; Philippova, O. E. Viscoelastic Synergy and 
Microstructure Formation in Aqueous Mixtures of Nonionic Hydrophilic Polymer and Charged Wormlike Surfactant Micelles. Macromolecules 2017, 50, 339-348.

(24) Raghavan, S. R.; Fritz, G.; Kaler, E. W. Wormlike Micelles Formed by Synergistic Self-Assembly in Mixtures of Anionic and Cationic Surfactants. Langmuir 2002, 18, 3797-3803.

(25) Ziserman, L.; Abezgauz, L.; Ramon, O.; Raghavan, S. R.; Danino, D. Origins of the Viscosity Peak in Wormlike Micellar Solutions. 1. Mixed Catanionic Surfactants. A Cryo-Transmission Electron Microscopy Study. Langmuir 2009, 25, 10483-10489.

(26) Kume, G.; Gallotti, M.; Nunes, G. Review on Anionic/Cationic Surfactant Mixtures. J. Surfactants Deterg. 2008, 11, 1.

(27) Koshy, P.; Verma, G.; Aswal, V. K.; Venkatesh, M.; Hassan, P. A. Viscoelastic Fluids Originated from Enhanced Solubility of Sodium Laurate in Cetyl Trimethyl Ammonium Bromide Micelles through Cooperative Self-Assembly. J. Phys. Chem. B 2010, 114, 10462-10470.

(28) Huang, J.; Zhang, S.; Feng, Y.; Li, J.; Yan, H.; He, F.; Wang, G.; Liu, Y.; Wang, L. Rheological Properties and Application of Wormlike Micelles Formed by Sodium Oleate/Benzyltrimethyl Ammonium Bromide. Colloids Surf., A 2016, 500, 222-229.

(29) Fukuda, H.; Goto, A.; Yoshioka, H.; Goto, R.; Morigaki, K.; Walde, P. An Electron Spin Resonance Study of the $\mathrm{pH}$-Induced Transformation of Micelles to Vesicles in an Aqueous Oleic Acid/ Oleate System. Langmuir 2001, 17, 4223-4231.

(30) Andreeva, A. S.; Philippova, O. E.; Khokhlov, A. R.; Islamov, A. K.; Kuklin, A. I. Effect of the Mobility of Charged Units on the Microphase Separation in Amphiphilic Polyelectrolyte Hydrogels. Langmuir 2005, 21, 1216-1222.

(31) Kuklin, A. I.; Soloviov, D. V.; Rogachev, A. V.; Utrobin, P. K.; Kovalev, Yu. S.; Balasoiu, M.; Ivankov, A. I.; Sirotin, A. P.; Murugova, T. N.; Petukhova, T. B.; Gorshkova, Yu. E.; Erhan, E. V.; Kutuzov, S. A.; Soloviev, A. G.; Gordeliy, V. I. New Opportunities Provided by Modernized Small-Angle Neutron Scattering Two-Detector System Instrument (YUMO). J. Phys.: Conf. Ser. 2011, 291, 012013.

(32) Soloviev, A. G.; Solovjeva, T. M.; Ivankov, O. I.; Soloviov, D. V.; Rogachev, A. V.; Kuklin, A. I. SAS Program for Two-Detector System: Seamless Curve from Both Detectors. J. Phys.: Conf. Ser. 2017, 848, 012020.

(33) Hayter, J. B.; Penfold, J. J. An Analytic Structure Factor for Macroion Solutions. Mol. Phys. 1981, 42, 109-118.

(34) Lutz-Bueno, V.; Liebi, M.; Kohlbrecher, J.; Fischer, P. Intermicellar Interactions and the Viscoelasticity of Surfactant Solutions: Complementary Use of SANS and SAXS. Langmuir 2017, 33, 2617-2627.

(35) Pedersen, J. S.; Cannavacciuolo, L.; Schurtenberger, P. Scattering from wormlike micelles. In Giant Micelles, Properties and Applications; Zana, R., Kaler, E. W., Eds.; CRC Press: Boca Raton, FL, 2007.

(36) Shibaev, A. V.; Molchanov, V. S.; Philippova, O. E. Rheological Behavior of Oil-Swollen Wormlike Surfactant Micelles. J. Phys. Chem. B 2015, 119, 15938-15946.

(37) Philippova, O. E.; Shibaev, A. V.; Muravlev, D. A.; Mityuk, D. Y. Structure and Rheology of Solutions and Gels of Stiff Polyelectrolyte at High Salt Concentration. Macromolecules 2016, 49, 6031-6040.

(38) Liu, C.; He, J.; van Ruymbeke, E.; Keunings, R.; Bailly, C. Evaluation of Different Methods for the Determination of the Plateau Modulus and the Entanglement Molecular Weight. Polymer 2006, 47, 4461-4479.

(39) Hebbeker, P.; Steinschulte, A. A.; Schneider, S.; Plamper, F. A. Balancing Segregation and Complexation in Amphiphilic Copolymers by Architecture and Confinement. Langmuir 2017, 33, 4091-4106.

(40) Lindman, B.; Khan, A.; Marques, E.; da Miguel, M. G.; Piculell, L.; Thalberg, K. Phase Behavior of Polymer-Surfactant Systems in Relation to Polymer-Polymer and Surfactant-Surfactant Mixtures. Pure Appl. Chem. 1993, 65, 953-958.

(41) Cates, M. E. Reptation of Living Polymers: Dynamics of Entangled Polymers in the Presence of Reversible Chain-Scission Reactions. Macromolecules 1987, 20, 2289-2296.
(42) Shibaev, A. V.; Tamm, M. V.; Molchanov, V. S.; Rogachev, A. V.; Kuklin, A. I.; Dormidontova, E. E.; Philippova, O. E. How a Viscoelastic Solution of Wormlike Micelles Transforms into a Microemulsion upon Absorption of Hydrocarbon: New Insight. Langmuir 2014, 30, 3705-3714.

(43) Flood, C.; Dreiss, C. A.; Croce, V.; Cosgrove, T.; Karlsson, G. Wormlike Micelles Mediated by Polyelectrolyte. Langmuir 2005, 21, $7646-7652$.

(44) Klymenko, A.; Nicolai, T.; Benyahia, L.; Chassenieux, C.; Colombani, O.; Nicol, E. Multiresponsive Hydrogels Formed by Interpenetrated Self-Assembled Polymer Networks. Macromolecules 2014, 47, 8386-8393.

(45) Sommer, C.; Pedersen, J. S.; Egelhaaf, S. U.; Cannavacciuolo, L.; Kohlbrecher, J.; Schurtenberger, P. Wormlike Micelles as "Equilibrium Polyelectrolytes": Light and Neutron Scattering Experiments. Langmuir 2002, 18, 2495-2505.

(46) Gamez-Corrales, R.; Berret, J.-F.; Walker, L. M.; Oberdisse, J. Shear-Thickening Dilute Surfactant Solutions: Equilibrium Structure as Studied by Small-Angle Neutron Scattering. Langmuir 1999, 15, $6755-6783$.

(47) Rehage, H.; Hoffmann, H. Viscoelastic Surfactant Solutions: Model Systems for Rheological Research. Mol. Phys. 1991, 74, 933973.

(48) Lequeux, F. Reptation of Connected Wormlike Micelles. Europhys. Lett. 1992, 19, 675-681.

(49) Khatory, A.; Kern, F.; Lequeux, F.; Appell, J.; Porte, G.; Morie, N.; Ott, A.; Urbach, W. Entangled versus Multiconnected Network of Wormlike Micelles. Langmuir 1993, 9, 933-939.

(50) Rogers, S. A.; Calabrese, M. A.; Wagner, N. J. Rheology of Branched Wormlike Micelles. Curr. Opin. Colloid Interface Sci. 2014, $19,530-535$.

(51) Kwiatkowski, A. L.; Molchanov, V. S.; Orekhov, A. S.; Vasiliev, A. L.; Philippova, O. E. Impact of Salt Co- and Counterions on Rheological Properties and Structure of Wormlike Micellar Solutions. J. Phys. Chem. B 2016, 120, 12547-12556.

(52) Colby, R. H. Structure and Linear Viscoelasticity of Flexible Polymer Solutions: Comparison of Polyelectrolyte and Neutral Polymer Solutions. Rheol. Acta 2010, 49, 425-442. 